\title{
Successful Transcatheter Closure of Patent Ductus Arteriosus in Isolated Levocardia with Situs Inversus
}

\author{
Adikesava Naidu Otikunta ${ }^{*}$, Y. V. Subba Reddy ${ }^{1}$, Sivaprasad Naidu Nallapati ${ }^{1}$, \\ S. A. A. Latheef ${ }^{2}$, Srinivas Ravi ${ }^{1}$ \\ ${ }^{1}$ Department of Cardiology, Osmania General Hospital \& Osmania Medical College, Hyderabad, India \\ ${ }^{2}$ School of Life Sciences, University of Hyderabad, Hyderabad, India \\ Email: "oadikesavanaidu@gmail.com
}

Received 12 April 2016; accepted 26 April 2016; published 29 April 2016

Copyright (C) 2016 by authors and Scientific Research Publishing Inc.

This work is licensed under the Creative Commons Attribution International License (CC BY).

http://creativecommons.org/licenses/by/4.0/

c) (i) Open Access

\begin{abstract}
Isolated levocardia with situs inversus is an extremely rare type of situs anomaly with an estimated incidence of 1 per 22,000 in the general population. This autosomal recessive situs anomaly has been described as normal levo position of the heart with dextro position of the abdominal viscera. In this case report, we describe accidental diagnosis of isolated levocardia with situs inversus in pediatric patient while evaluating for heart murmur. Systematic examination of the patient identified the presence of patent ductus arteriosus, anomalous course of inferior vena cava and bovine aortic arch. We shared our experience of successful transcatheter closure of patent ductus arteriosus. This case report is worth reporting both, for demonstrating the possibility of the percutaneous device closure of the patent ductus arteriosus in patient with this unusual situs anomaly to interventional cardiologists and because of scanty of literature.
\end{abstract}

\section{Keywords}

Situs Inversus, Isolated Levocardia, Patent Ductus Artrious, Transcatheter Closure, Anomalous Inferior Vena Cava, Bovine Aortic Arch

\section{Introduction}

The term "Situs" is used to define position of asymmetric organs of the human body relative to the midline. The usual orderly arrangement of asymmetric organs is refereed as situs solitus [1]. However, the failure of the nor-

\footnotetext{
*Corresponding author.
}

How to cite this paper: Otikunta, A.N., Y.V. Subba Reddy, Nallapati, S.N., Latheef, S.A.A. and Ravi, S. (2016) Successful Transcatheter Closure of Patent Ductus Arteriosus in Isolated Levocardia with Situs Inversus. World Journal of Cardiovascular Diseases, 6, 88-93. http://dx.doi.org/10.4236/wjcd.2016.64010 
mal development of asymmetry across the left-right axis leads to situs anomalies. Situs inversus (SI), an autosomal recessive situs anomaly, was first described by Matthew Baillie in 1788 [2] [3]. When the heart is located to the right side of the thorax and atria and the ventricles are inverted in accordance with the inverted viscera, the condition is known as "situs inversus with dextrocardia" or "situs inversus totalis". However, if the heart is in the normal position (levo) but the abdominal viscera are transposed laterally, it is known as isolated levocardia with SI (or SI with levocardia) [4].

Isolated levocardia with SI is an extremely rare situs anomaly. The estimated incidence is 1 per 22,000 in the general population [5] [6]. The published data suggest higher prevalence of congenital cardiac anomalies in patients with isolated levocardia with SI. Thus, this situs anomaly is usually identified at birth or soon afterwards if associated congenital cardiac anomalies are severe and complex [7] [8]. However, it may be a chance discovery during regular check-up or if the patient presents with untraditional presentation of referred pain (if the absence of severe and complex congenital cardiac anomaly) [5] [6] [9] [10]. Here, we present a case of a pediatric patient who was fortuitously found to have isolated levocardia with SI while evaluating for heart murmur. As the patient was diagnosed with patent ductus arteriosus (PDA), we performed transcatheter closure of PDA. One year follow-up of the patient was satisfactory without any residual shunt across PDA. To the best of our knowledge, this is the first case-report which demonstrated successful transcatheter closure of PDA in pediatric patient with situs anomaly. Moreover, the combination of presence of bovine aortic arch, anomalous course of inferior vena cava (IVC) and PDA in patient with isolated levocardia with SI has not been reported so far in the literature.

\section{Case Report}

A 7 year-old-female child was referred to our clinic for the evaluation of heart murmur by the pediatrician. She had fever associated with upper respiratory tract infection which was subsided with treatment. Her past medical history was unremarkable except recurrent respiratory tract infections.

The patient was a moderately built and nourished child. There was no evidence of pallor, icterus, clubbing, cyanosis, lymphadenopathy or edema. The patient had a regular, high volume pulse rate of 100/min (without radio-radial or radio-femoral delay) and normal jugular venous pulse. However, we found hyperdynamic apical impulse in left fifth intercoastal space, lateral to mid clavicular line in addition to systolic thrill at left second and first intercostal space. There was a continuous murmur (grade 4/6) of rough machinery character in left second and first intercoastal spaces and early systolic murmur of mixed frequency at second intercoastal space radiated to both carotids with early peaking. We also noticed aortic regurgitation. However, the blood pressure recording of all the limbs (right upper limb: 130/40 mm Hg; left upper limb: 130/40 mm Hg; and right lower limb: 150/40 mm Hg) ruled out the presence of coarctation of aorta which is commonly associated with PDA. We noticed absence of normal liver dullness on right. While percussion of the abdomen, we found liver border on left fifth intercostal space and stomach on right side.

A posteroanterior chest radiograph demonstrated normal position of the heart, cardiomegaly, pulmonary plethora and right-sided gastric air bubbles (Figure 1). An electrocardiogram (ECG) indicated left ventricle (LV)

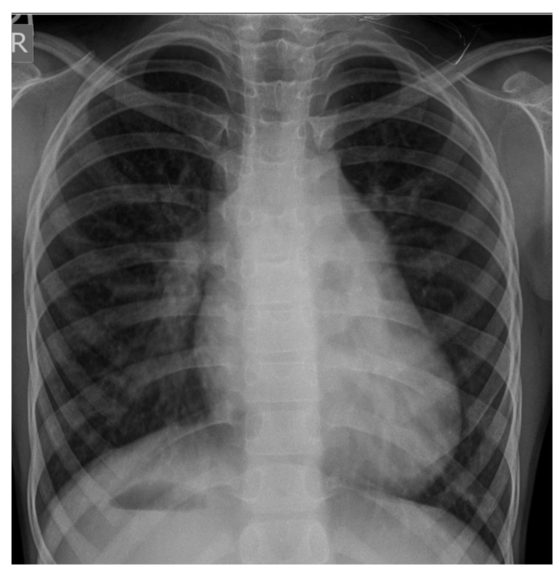

Figure 1. Chest X-ray showing cardiomegaly with pulmonary plethora and right-sided gastric air bubbles. 
volume overload pattern (Figure 2). Two-dimensional echocardiography confirmed the presence of PDA (8 $\mathrm{mm}$ ), dilated left ventricle and left atrium due to left to right shunt (>2:1) (Figure 3(A) \& Figure 3(B)). Apart from PDA, echocardiogram also revealed the presence of aorta and IVC on left side of spine, anomalous course of IVC (left side of aorta) and mild aortic regurgitation without aortic stenosis (Figure 3(C)). There was a concordant atrio-ventricular and ventriculo-arterial connection. Based upon the findings of echocardiography and physical examinations, we suspected the possibility of isolated levocardia with SI and PDA.

Computed tomography (CT) of chest and CT angiography also indicated isolated levocardia with SI along with anomalous course of IVC (lie to the left of aorta and entered right atrium after crossing aorta from left to right) (Figure 4(A) \& Figure 4(B)) and bovine aortic arch (Figure 4(C)). However, other abnormalities were not found. We confirmed the diagnosis of isolated levocardia with SI, anomalous course of IVC, bovine aortic arch and PDA.

We decided to perform transcatheter closure of PDA through right femoral approach as pulmonary to systemic flow ratio $(\mathrm{Qp} / \mathrm{Qs})$ was 2:1 and no pulmonary hypertension. An aortic angiogram was performed in lateral view to demonstrate PDA by placing 6F pig tail beyond the ductus in the aorta. We observed left sided aortic arch, PDA with well-defined aortic ampulla and constriction near the pulmonary artery end. Through femoral venous approach, we tried to cross PDA using $6 \mathrm{~F}$ Cournand catheter as well as multi-purpose catheter. Finally LIMA catheter has been advanced anterogradely from the femoral vein and through the duct into the descending aorta. LIMA catheter is then exchanged for a 7F PDA delivery sheath, over a 0.035 " exchange length amplatz guidewire with the tip of the sheath in the descending aorta. An occluder device $(10 \mathrm{~mm} \times 12 \mathrm{~mm})$, CERA ${ }^{\mathrm{TM}}$ (Lifetech Scientific Co Ltd., Shenzhen, China) PDA occluder, was deployed across the PDA with delivery system after confirming absence of residual shunt.

There was no post-procedural complication and echocardiogram also showed no residual shunt (Figure 5).
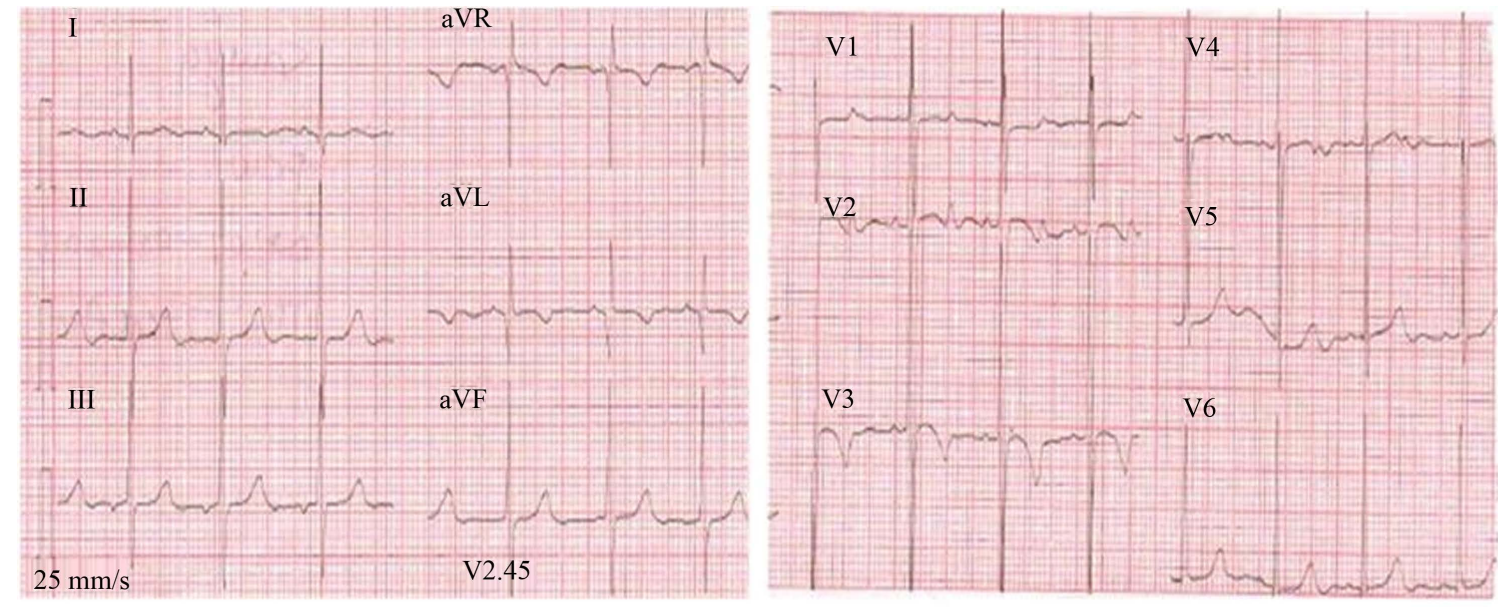

Figure 2. Electrocardiogram showing left ventricular volume overload pattern.
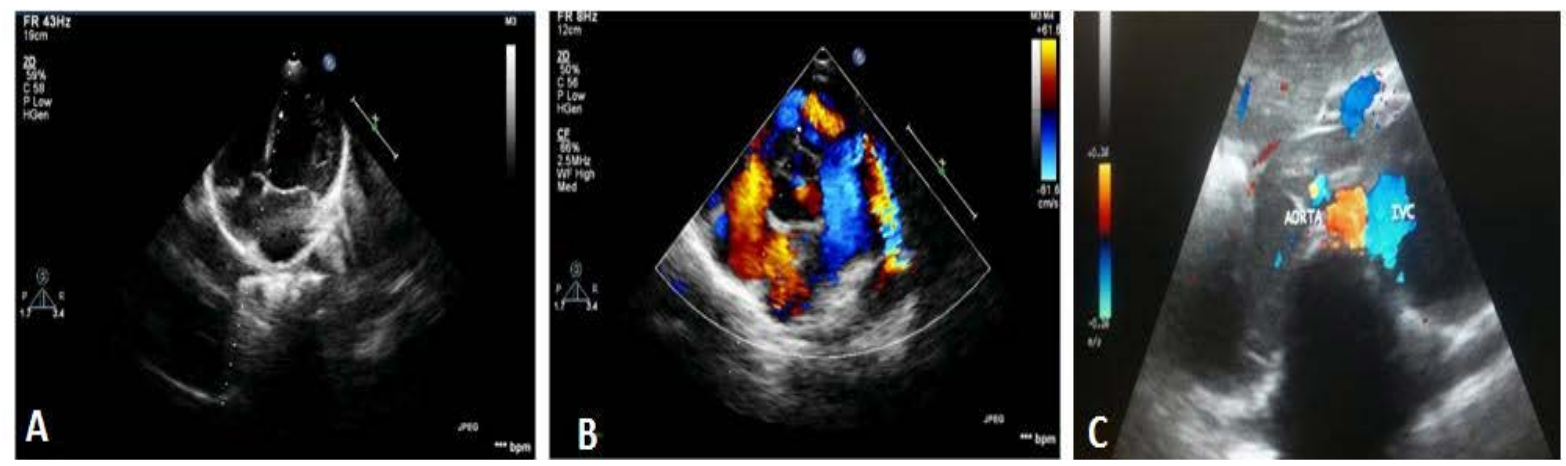

Figure 3. (A) Echocardiogram in 4 chamber view showing dilated left ventricle; (B) Color Doppler Echocardiogram in short axis view showing patent ductus arteriosus; (C) Echocardiogram showing inferior vena cava lie to the left of aorta. 

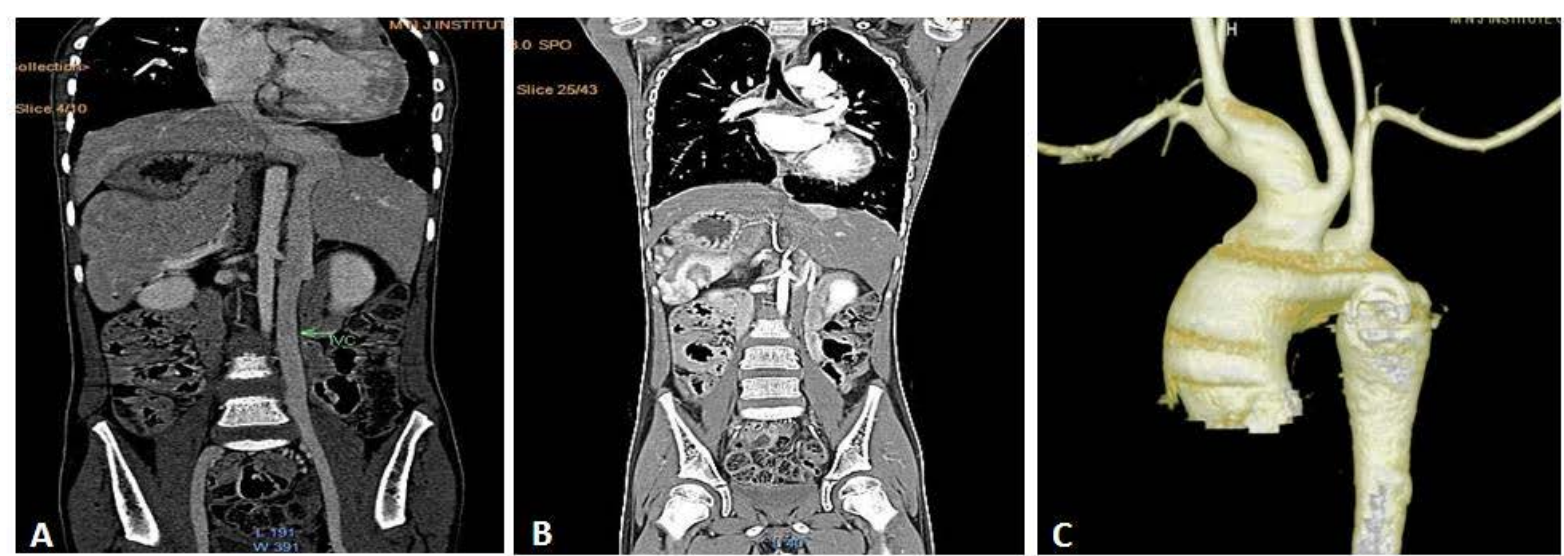

Figure 4. (A) \& (B) Computed Tomography scan reconstructed film showing anomalous course of inferior vena cava which lie left side of aorta entering into right atrium crossing from left to right and abdominal situs inversus; (C) Computed Tomography Angiography showing bovine aortic arch.

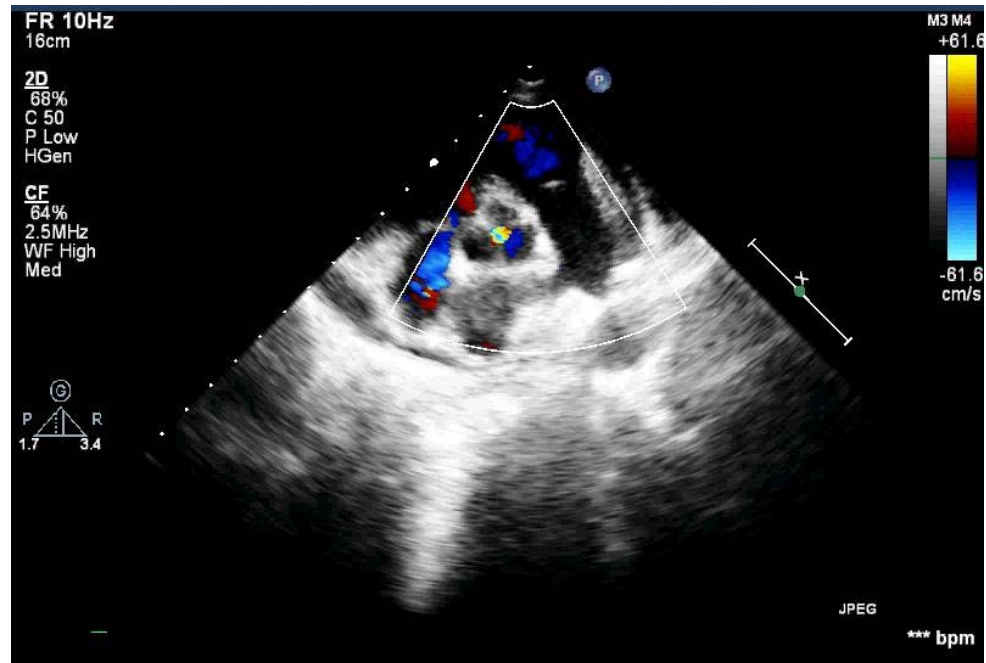

Figure 5. Post-procedural echocardiogram showing patent ductus arteriosus closure with no residual shunt.

One-year follow up was satisfactory with no residual shunt across PDA, decreasing size of LV as well as normal $\mathrm{LV}$ function. The parents of the patient had given consent for publication of case report.

\section{Discussion}

In humans, asymmetry along the left-right axis is determined at the beginning of the embryonic development. At approximately 23 days of gestation, the primitive heart tube initially bends to the right in a dextral-loop (d-loop) to place the right ventricle and then moves to the left until the ventricular portion occupies a normal left thoracic position [1] [11]. The bend of the cardiac tube to right is the first sign of the asymmetry. Recent research has shown that mutations in the genes which are involved in the determination of the left-right asymmetry, for example lefty, nodal, inversus viscerum (iv), HAND, ZIC3, sonic hedgehog (Shh), ACVR2B and Pitxz, are implicated in situs anomalies [11]. In addition to SI, situs ambigus is also recognized as a type of situs anomaly which is characterized by absence of asymmetric organ lateralization [1].

Approximately $0.4 \%$ to $1.2 \%$ patients with congenital heart disease are found to have isolated levocardia with SI. Several previous case-reports documented severe and complicated cardiac malformation in such patients. Young and Griswold reported case-series of 8 patients who were identified to have isolated levocardia with SI [7]. The reported congenital cardiac malformations in these patients include transposition of great vessels, pulmonary stenosis, ventricular septal defect, PDA, patent foramen ovale, dextroposition of aorta, pulmonary atre- 
sia, anomalous superior vena cava and anomalies of the pulmonary venous return. Cyanotic congenital heart diseases (transposition of atria, ventricular septal defect, atrial septal defects and diminutive right ventricle) in an infant were reported by Padmavati et al. [8] However, literature search also showed case-reports in which patients with isolated levocardia with SI did not have any congenital heart disease [6] [10]. In our patient, multiple diagnostic procedures identified presence of type-A (conical) PDA and ruled out complex cardiac malformation such as ventricular septal defect, atrial septal defect, pulmonary atresia, pulmonary stenosis, bilateral superior vena cava, left ventricular out flow tract obstruction, tetralogy of fallot, truncus arteriosus and transposition of great vessels. However, it is imperative to be cognizant of the anomalous anatomy before planning interventions to ensure safety and avoid complications in patients with isolated levocardia with SI. Thus, we performed CT-scan and CT angiography in our patient to identify such abnormal anatomy of vasculature as conventional means often fails to describe diverse spectrum of anomalies in such patients. The preoperative image workup in our patient helped us to identify anomalous course of IVC and bovine aortic arch. Anomalous IVC in patients with isolated levocadia with SI has been reported in previously published case-reports. Gindes et al. performed prenatal and postnatal echocardiography in 3 fetuses with isolated levocardia [12]. The result of the study showed left-sided IVC in one fetus and anomalous IVC in two fetuses (intrahepatic interruption of IVC and azygous continuation of IVC). The authors reported uneventful long-term survival of all the fetuses. Sacks et al. also reported interruption of IVC with azygous continuation (at the level of L1) in a 20-year old male patient who was accidentally found to have situs anomaly with left sided morphologically normal heart and mirror image arrangement of abdominal organs [10]. Azygous continuation of IVC was also reported by Vijaykumar et al. in 73-year old patient [6]. However, in our patient, IVC lie to the left of of aorta and entered right atrium after crossing aorta from left to right. Thus, the course of IVC was different. There was no evidence of interruption of IVC.

Bovine aortic arch is the most common variant of arch anatomy which is the result of slow growth of the ventral aortic roots between third and fourth embryological arches. it is seen in $10 \%-20 \%$ of the population [13]. This anatomic configuration of aortic arch refers to both shared origin of the left common carotid and innominate arteries, and origin of the left common carotid artery from the innominate artery. In our case, CT angiography showed bovine aortic arch in which left common carotid artery originated from the innominate artery. Though the presence of bovine aortic arch did not make any difference regarding PDA intervention, the interventional cardiologists should note that PDA in such patients may not be crossed with routine catheters.

\section{Conclusion}

In conclusion, we shared our experience of successful transcatheter closure of PDA in pediatric patient with isolated levocardia with SI. This case-report is worth reporting both, for demonstrating the possibility of the percutaneous device closure of the PDA in such patient to interventional cardiologists and because of scanty of literature related to this unusual situs anomaly.

\section{References}

[1] Perloff, J.K. (2011) The Cardiac Malpositions. American Journal of Cardiology, 108, 1352-1361. http://dx.doi.org/10.1016/j.amjcard.2011.06.055

[2] Baillie, M. (1788) An Account of a Remarkable Transposition of the Viscera. Philosophical Transactions of the Royal Society A, 78, 350-363. http://dx.doi.org/10.1098/rstl.1788.0023

[3] Yokoyama, T., Copeland, N.G., Jenkins, N.A., Montgomery, C.A., Elder, F.F. and Overbeek, P.A. (1993) Reversal of Left-Right Asymmetry: A Situs Inversus Mutation. Science, 260, 679-682. http://dx.doi.org/10.1126/science.8480178

[4] Perloff, J.K. and Marelli, A.J. (2012) Clinical Recognition of Congenital Heart Disease. 6th Edition, Elsevier Health Sciences, 368-394.

[5] Budhiraja, S., Singh, G., Miglani, H.P. and Mitra, S.K. (2000) Neonatal Intestinal Obstruction with Isolated Levocardia. Journal of Pediatric Surgery, 35, 1115-1116. http://dx.doi.org/10.1053/jpsu.2000.7839

[6] Vijayakumar, V. and Brandt, T. (1991) Prolonged Survival with Isolated Levocardia and Situs Inversus. Cleveland Clinic Journal of Medicine, 58, 243-247. http://dx.doi.org/10.3949/ccjm.58.3.243

[7] Young, M.D. and Griswold, H.E. (1951) Situs Inversus of the Abdominal Viscera with Levocardia; Report of Eight Cases Submitted to the Blalock-Taussig Operation. Circulation, 3, 202-214. http://dx.doi.org/10.1161/01.CIR.3.2.202

[8] Padmavati, S. and Gupta, S. (1962) Partial Situs Inversus with Levocardia. An Unusual Combination of Anomalies. 
Circulation, 26, 108-113. http://dx.doi.org/10.1161/01.CIR.26.1.108

[9] Tryfonas, G.I., Chaidos, C., Avtzoglou, P.P., Zioutis, J., Klokaris, A. and Papanastasopoulos, A. (1992) Partial Situs Inversus: Duodenal Obstruction in a Neonate with Isolated Levocardia. Journal of Pediatric Surgery, 27, 1584-1586. http://dx.doi.org/10.1016/0022-3468(92)90517-B

[10] Sacks, L.V. and Rifkin, I.R. (1987) Mirror Image Arrangement of the Abdominal Organs with a Left-Sided Morphologically Normal Heart. British Heart Journal, 58, 534-536. http://dx.doi.org/10.1136/hrt.58.5.534

[11] Bowers, P.N., Brueckner, M. and Yost, H.J. (1996) Laterality Disturbances. Progress in Pediatric Cardiology, 6, 53-62. http://dx.doi.org/10.1016/1058-9813(96)00171-3

[12] Gindes, L., Hegesh, J., Barkai, G., Jacobson, J.M. and Achiron, R. (2007) Isolated Levocardia: Prenatal Diagnosis, Clinical Importance, and Literature Review. Journal of Ultrasound in Medicine, 26, 361-365.

[13] Malone, C.D., Urbania, T.H., Crook, S.E. and Hope, M.D. (2012) Bovine Aortic Arch: A Novel Association with Thoracic Aortic Dilation. Clinical Radiology, 67, 28-31. http://dx.doi.org/10.1016/j.crad.2011.04.004 